\title{
Alterations of HLA class I and II antigen expression in preinvasive, invasive and metastatic cervical cancers
}

\author{
Ki Sung Ryu' ${ }^{1^{\star}}$, Youn Soo Lee ${ }^{2^{\star}}$, Byung Kee Kim², \\ Yong Gyu Park ${ }^{3}$, Yong Wook Kim ${ }^{1}$, \\ Soo Young Hur ${ }^{1}$, Tae Eung Kim ${ }^{1}$, \\ In-Kyung Kim ${ }^{4}$ and Jin Woo Kim ${ }^{1,5,6}$

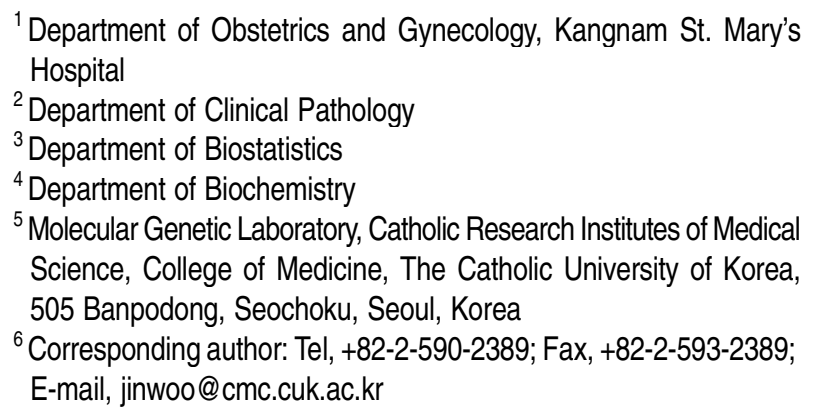

Accepted 12 September, 2001

Abbreviations: CIS, carcinoma in situ; MIC, microinvasive carcinoma; SCC, squamous cell carcinoma; MHC, major histocompatibility complex; TAP, transporter associated with antigen presentation; mAb, monoclonal antibody; $\beta 2 \mathrm{~m}, \beta 2$-microglobulin; IHS, Immunohistochemical staining; H\&E, hematoxylin-eosin

\begin{abstract}
HLA expression is altered in a large variety of human cancers. We performed immunohistochemical staining on tissues from normal cervix, preinvasive, invasive and metastatic cervical cancer using anti-HLA class I or class II antibody. In tissues from normal squamous epithelium, carcinoma in situ (CIS) and microinvasive carcinoma (MIC), the expressions of HLA-B, C heavy chains and class II heavy chain were significantly decreased as disease progressed. When the expression patterns were compared between primary and metastatic squamous cell carcinoma (SCC) lesions, statistically significant down-regulations of HLA class I and class II antigen in metastatic lesions were observed. The rates of HLA-B, C heavy chains and class II heavy chain expressions were all significantly down-regulated compared to the down-regulation rate of class I $\beta 2$-microglobulin $(\beta 2 \mathrm{~m})$ in invasive squamous lesions 'The expression of class II heavy chain in metastatic lesions was decreased further than that in primary lesions. Unlike SCC, the changes of HLA class I and class II expression were not evident,
\end{abstract}

however in early stage of adenocarcinoma. In invasive adenocarcinoma lesions, only the expression of HLA-B, $C$ heavy chains was decreased no difference was seen in HLA-B, $C$ heavy chain expression pattern between primary and metastatic lesions. These results suggest that alterations of HLA class I and II expressions seem to occur at a particular step in cervical cancer progression and depend on tissue types: when the tumor becomes invasive and starts to metastasize.

Keywords: cervical cancer, HLA class I, HLA class II, IHS, metastasis

\section{Introduction}

The major histocompatibility complex (MHC) comprises a family of highly polymorphic genes encoding a set of transmembrane proteins that present peptide epitopes to specific antigen receptors on T cells (Howard et al., 1999). These antigens eventually are reduced to small peptides in the endoplasmic reticulum of the cell and transported to the cell membrane together with a particular $\mathrm{MHC}$ allele to interact with a T-cell receptor-CD3 complex (Townsend et al., 1989). Four major categories of genes are involved in this genetically controlled process: HLA class I, HLA class II, proteasome, and transporter genes (Garrido et al., 1995). A major characteristic of these genes is their very high degree of HLA polymorphism (Campbell and Trowsdale, 1993). In humans, polymorphism is concentrated mainly in class I and class II genes (Powis et al., 1992).

The down-regulation of all or some HLA alleles in a particular individual by various causes breaks the polymorphism and abolishes the capacity to present antigens through $\mathrm{MHC}$ products. Cell transformation is associated in many cases with MHC down-regulation (Schrier et al., 1983). Altered HLA phenotypes could arise during any of the different steps in the biosynthetic pathway leading to HLA membrane expression, i.e. transcription-regulation defects, overexpression of protooncogene products, loss of transporter molecules, mutations in or loss of $\beta 2 \mathrm{~m}$ gene or HLA genes, and loss of HLA haplotypes secondary to loss of heterozygosity (Browning and Dunnion, 1979; Garrido et al., 1997; Koopman et al., 1998).

In cervical cancer, causes which may contribute to HLA down-regulation have been scantily studied. Decreased HLA expression was not correlated with the presence of 
human papillomavirus 16 or 18 in cervical cancers (Cromme et al., 1993a). It has been suggested that down-regulation of class I MHC expression in cervical cancers is apparently post-transcriptionally controlled (Cromme et al., 1993b). This was consistent with the observation that class I MHC loss is frequently associated with a failure to detect one of the subunits of the transporter associated with antigen presentation (TAP-1) (Cromme et al., 1994a). Yet, deficiencies in TAP were correlated with only a portion of HLA class I downregulated tumors (Keating et al., 1995). However, little is known about the biological behaviour of cervical cancers in association with down-regulation of class I and II MHC. Up-regulated HLA-DR expression was found exclusively in primary cervical cancers and was absent in the corresponding metastasis (Cromme et al., 1994b). Higher frequency of selective losses of HLA-A and -B allospecificities was observed in metastasis than in primary lesions (Geertsen et al., 1998). These findings suggest that selective loss of HLA class I or II allospecificities might be a major step in tumor progression.

In this study, we investigated whether immunohistochemically determined changes in class I and II MHC expression are related to tumor progression in human cervical cancer tissues.

\section{Materials and Methods}

\section{Study subjects and tissue sampling}

Paraffin-embedded tissue specimens were obtained from patients with cervical carcinoma who were treated at the Kangnam St. Mary's Hospital from 1994 to 1998. From all primary tumors and regional lymph node metastases, representative paraffin blocks were selected and serially cut to 4 um sections. To ensure the presence of tumor, the first and last slide from each series were stained routinely with hematoxylin-eosin $(H$ \& $E)$ and subjected to microscopic examination. The number of the subjects was 21 cases of microinvasive SCC with CIS component, 26 cases of invasive SCC, 3 cases of microinvasive adenocarcinoma with CIS component, and 9 cases of invasive adenocarcinoma. Among invasive carcinoma cases, 25 of 26 SCCs and 5 of 9 adenocarcinomas showed regional lymph node metastasis.

\section{Monoclonal antibodies}

We used the monoclonal antibody (mAb) L368 for $\beta 2 m$ of HLA class I, the mAb HC-10 for HLA-B, C heavy chains, and the mAb LGII-612.14 for HLA class II heavy chain. $L 368$ is a mouse $\operatorname{lgG}_{1}$ that recognizes $\beta 2 \mathrm{~m}, \mathrm{HC}-10$ is a mouse $\operatorname{lgG}_{2}$ that recognizes common epitope of HLA-B, C heavy chains, and LGII-612.14 is an antibody that recognizes the monomorphic deter- minant of $\beta$ chain of HLA-DR, -DQ, and DP (Temponi et al., 1993). Monoclonal antibodies were kindly provided by Dr. Soldano Ferrone (Rosewell Park Cancer Institute, Buffalo, New York).

\section{Immunohistochemical staining (IHS)}

We used the avidin-biotin-peroxidase complex method (Hsu et al., 1981). The paraffin-embedded tissues were dewaxed using xylene, treated with graded ethanol, rehydrated after removing paraffin and washed with water. Then, the tissues were placed in peroxide quenching solution made with hydrogen peroxide for $30 \mathrm{~min}$ to remove the endogenous peroxidase activity, treated with serum blocking solution (Zymed Laboratories, CA) for 30 min to block non-specific binding, treated with the primary antibody, left for overnight at $4^{\circ} \mathrm{C}$, washed with phosphate-buffered saline (PBS), treated with the biotinylated secondary antibody (Zymed) for $30 \mathrm{~min}$, and washed with PBS. Then, the tissues were treated with an enzyme conjugate for $30 \mathrm{~min}$, washed with PBS, treated with the aminoethyl carbazole (Zymed) for $18 \mathrm{~min}$ as a chromogen. Then, the tissue sections were counterstained with hematoxylin.

\section{Analysis of HLA expression and statistics}

Ubiquitously present class I or II positive stromal cells (lymphocytes, fibroblasts, vascular endothelial cells, etc) and normal cervical epithelium served as intrinsic positive controls for the presence of class I or II specificity in a given tissue and for the strength of the immunoreactivity of the mAb used. The intensity of staining was evaluated semiquantitatively and divided into 4 grades as follows: ++ , strong positive; + , moderately positive; \pm , weakly positive; -, negative. In case of heterogeneously stained tumors, corresponding tumor cell clusters were compared in the sequence of sections. If possible, we tried to make the reading to include one entire epithelial layer for normal cervical epithelium and one entire layer for cancer tissues.

For the purpose of statistical analyses, the expression rates were converted into a 4-point system in which 1 point was given to $(-)$ reading, $2( \pm), 3(+)$ and $4(++)$ according to the examined changes in HLA expression patterns. The converted points of HLA expression were examed statistically and presented in mean, median, and range between minimum and maximum values.

The relationship between the expression rate of HLA and histological type of the tissue was examined by Wilcoxon signed rank test and the differences amongst HLA expression patterns using each mAb were compared by Friedman test and Millers nonparametric multiple comparison. $P$ values less than 0.05 were considered statistically significant. To compare the differences between primary and metastasis by matched sample analysis, we excluded the non-metastatic cases. 


\section{Results}

HLA expression patterns in normal, CIS and microinvasive SCCs

HLA class I and II expression patterns in CIS and microinvasive SCC lesions were measured and compared with those in normal cells present in the tissue section (Table 1). Table 2 shows the degree of HLA expression expressed in mean, median and range of minimum to maximum value in each tissue. When Friedman test was used for analysis, the expression patterns of HLA class I and II all showed significant differences among normal, CIS, microinvasive SCC tissues whereas there were no significant differences between each two tissues (normal versus CIS; CIS versus microinvasion; microinvasion versus normal) in HLA class I $\beta 2 m$ expression pattern, compared by Miller's nonparametric multiple comparison. However, the expressions of HLA-B, C heavy chains and class II heavy chain all showed significant down-regulation in CIS and microinvasive tissues compared to normal tissue $(P=0.0001)$. Also, the expressions of HLA-B, C heavy chains $(P<0.05)$ and class II heavy chain $(P<0.05)$ were significantly down-regulated in microinvasive tissues compared to CIS tissues. Degree of down-regulation from normal tissues to CIS and from CIS to microinvasion, respectively, in HLA class I $\beta 2 \mathrm{~m}$ was all significantly lower than those in HLA-B, $C$ heavy chains or class II heavy chain $(P<0.05)$.

IHS using HC-10 mAb revealed that the degree of

Table 1. Staining patterns for HLA class I and II in normal, CIS and MIC

\begin{tabular}{|c|c|c|c|c|c|c|c|c|c|}
\hline \multirow{2}{*}{ Patient } & \multicolumn{3}{|c|}{ L368 mAb } & \multicolumn{3}{|c|}{$\mathrm{HC} 10 \mathrm{mAb}$} & \multicolumn{3}{|c|}{ LGII-612.14 mAb } \\
\hline & MIC & CIS & Normal & MIC & CIS & Normal & MIC & $\mathrm{CIS}$ & Normal \\
\hline 1 & \pm & \pm & ++ & \pm & \pm & ++ & \pm & \pm & ++ \\
\hline 2 & + & + & + & \pm & \pm & \pm & ++ & + & ++ \\
\hline 3 & + & + & + & + & + & + & + & + & ++ \\
\hline 4 & + & ++ & ++ & \pm & + & + & \pm & + & ++ \\
\hline 5 & \pm & \pm & \pm & + & + & ++ & \pm & \pm & + \\
\hline 6 & ++ & ++ & ++ & + & + & ++ & \pm & \pm & ++ \\
\hline 7 & + & + & + & + & + & ++ & ++ & ++ & ++ \\
\hline 8 & \pm & \pm & + & \pm & \pm & ++ & - & - & ++ \\
\hline 9 & + & + & + & + & + & ++ & \pm & \pm & ++ \\
\hline 10 & \pm & \pm & \pm & \pm & \pm & \pm & \pm & \pm & ++ \\
\hline 11 & + & + & + & \pm & \pm & ++ & + & + & ++ \\
\hline 12 & \pm & \pm & ++ & \pm & \pm & ++ & + & + & ++ \\
\hline 13 & \pm & \pm & + & - & - & ++ & \pm & \pm & ++ \\
\hline 14 & ++ & ++ & ++ & + & ++ & ++ & + & + & ++ \\
\hline 15 & + & + & + & ++ & ++ & ++ & \pm & \pm & ++ \\
\hline 16 & \pm & \pm & ++ & \pm & \pm & ++ & ++ & ++ & ++ \\
\hline 17 & + & + & + & ++ & ++ & ++ & + & ++ & ++ \\
\hline 18 & + & + & + & \pm & \pm & ++ & + & + & + \\
\hline 19 & + & + & + & ++ & ++ & ++ & \pm & \pm & + \\
\hline 20 & \pm & \pm & \pm & \pm & \pm & ++ & + & + & ++ \\
\hline 21 & + & + & + & + & + & ++ & + & + & ++ \\
\hline
\end{tabular}

Semiquantitative evaluation of immunoreactivity of normal and tumor cells are described in Materials and Methods.

Table 2. HLA class I and II expressions in early stage of SCC

\begin{tabular}{|c|c|c|c|c|c|c|c|c|c|c|c|c|}
\hline & \multicolumn{4}{|c|}{ L368 mAb } & \multicolumn{4}{|c|}{$\mathrm{HC} 10 \mathrm{mAb}$} & \multicolumn{4}{|c|}{ LGIl-612.14 mAb } \\
\hline & No. & Mean & Median & Range & No. & Mean & Median & Range & No. & Mean & Median & Range \\
\hline MIC & 21 & 2.714 & 3 & $2-4$ & 21 & $2.571^{\star \dagger}$ & 2 & $1-4$ & 21 & $2.667^{* \dagger}$ & 3 & $1-4$ \\
\hline CIS & 21 & 2.761 & 3 & $2-4$ & 21 & $2.667^{\star \ddagger}$ & 3 & $1-4$ & 21 & $2.714^{\star \star}$ & 3 & $1-4$ \\
\hline Normal & 21 & 3.142 & 3 & $2-4$ & 21 & 3.714 & 4 & $2-4$ & 21 & 3.857 & 4 & $3-4$ \\
\hline$P$-value & \multicolumn{4}{|c|}{0.035} & \multicolumn{4}{|c|}{0.001} & \multicolumn{4}{|c|}{0.001} \\
\hline
\end{tabular}

$P$-value; comparing the difference among three tissues in each class by Freidman test.

*denotes significant difference compared to normal epithelial tissue by Miller's nonparametric multiple comparison $(P<0.05)$.

${ }^{\dagger}$ denotes significant difference compared to L368 in the degree of down-regulation from CIS by Friedman test and Miller's nonparametric multiple comparison $(P$ $<0.05$ ).

denotes significant difference compared to L368 in the degree of down-regulation from normal epithelial tissue by Friedman test and Miller's nonparametric multiple comparison $(P<0.05)$. 

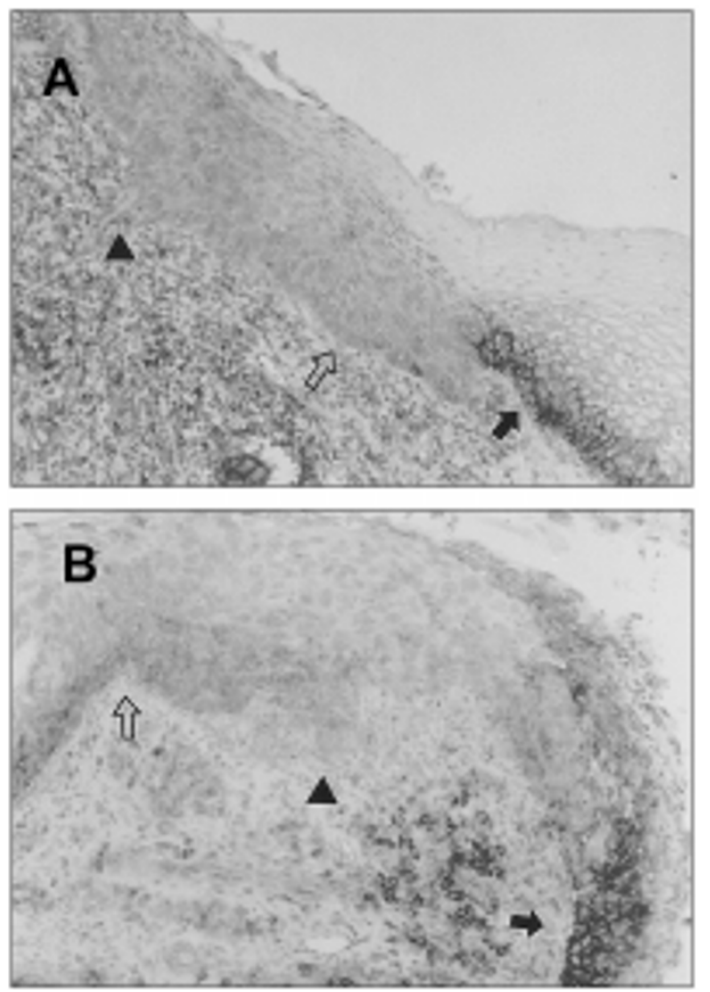

Figure 1. Immunohistochemical staining of early stage of SCC. (A) HC-10 mAb against HLA-B, C heavy chains was used. Left middle one third of atypical squamous cells is suspicious of the early stromal invasion and show negative intensity of expression (arrowhead). The adjacent CIS lesion shows weak positive intensity of expression (open arrow). The normal epithelial tissue, right middle one third with intact basement membrane shows strong positive intensity of expression compared with the CIS and invasive lesion definitely (closed arrow). Note the intense stromal lymphocytic infiltration around the invasion area. Original magnification. $\times 100$. (B) HLA class II heavy chain expression in early stage of SCC. Right lower normal epithelial tissue with intact basement membrane shows strong positive expression (closed arrow). Left upper CIS lesion with intact basement membrane shows moderate positive intensity of expression (open arrow). The central portion shows basement membrane disruption with early stromal invasion (arrowhead). This lesion expresses weak positive intensity and focally negative intensity of expression. (Magnification $\times 100$ ).

HLA-B, C heavy chain loss was evident as disease progressed (Figure 1A). The normal tissue with intact basement membrane shows definitive strong positive intensity of expression compared with the CIS and microinvasion lesions. Early stromal invasion lesion showed negative intensity of expression and the adjacent CIS lesion showed weak positive and focally negative intensity of expression.

IHS using LGII-612.14 mAb against HLA class II heavy chain showed similar staining pattern like $\mathrm{HC}-10$ mAb (Figure 1B).

\section{Changes in HLA expression patterns according to the progression of SCC}

HLA class I and II expression patterns in primary invasive cervical cancer tissues and metastatic lesions were measured and compared with that in normal cells present in the tissue section (Table 3). Table 4 shows the degree of HLA expression expressed in mean, median and range of minimum to maximum value in each tissue.

After determining the patterns of HLA expressions in primary and metastatic lesions of invasive SCC, the degrees of HLA expression of tumor tissues and corresponding normal stromal tissues were compared. According to Table 4, in primary and metastatic lesions of invasive SCC, the HLA class I and class II expressions all showed statistically significant down-regulation in primary (Figures 2A, C) and metastatic (Figures 2B, D) lesions compared to those in normal stromal tissues $(P<0.05)$.

Also, when the expression patterns were compared between primary and metastatic lesions, statistically significant down-regulations of HLA class I (Figure 2B) and class II (Figure 2D) in metastatic lesions were seen $(P<0.05)$.

However, the HLA expression patterns of normal stromal tissues in primary and metastatic lesions did not show the significant differences. When Friedman test and Miller's nonparametric multiple comparison were performed to compare the down-regulation rates from normal stromal tissues to tumor tissues among HLA class I and II expressions, the results showed that the down-regulation rate of class II heavy chain expression was significantly higher than that of class $\mathrm{I} \beta 2 \mathrm{~m}$ in primary lesions $(P<0.05)$. However, there was no significant difference between the down-regulation rates of class I $\beta 2 m$ and HLA-B, C heavy chains. Also, in metastatic lesions, the rates of HLA-B, $C$ heavy chains and class II heavy chain expressions were all significantly down-regulated compared to the down-regulation rate of class I $\beta 2 \mathrm{~m}(P<0.05)$.

Thus, when HLA classes were compared in invasive SCC, the down-regulation rates of HLA-B, C heavy chains and class II heavy chain were significantly higher than that of HLA class I $\beta 2 \mathrm{~m}$. Metastatic lesion shows strong negative expression compared with the primary lesion from the same patient.

\section{HLA expression patterns in normal, CIS and micro- invasive adenocarcinomas}

HLA class I and II expression patterns in CIS and microinvasive adenocarcinoma lesions were measured and compared with those in normal cells present in the tissue section (Table 5). Table 6 shows the degrees of expressions in mean, median and range from minimum to maximum values according to HLA types.

In this study, the expressions of HLA class I and class II showed no difference among normal, CIS and microinvasive tissues $(P>0.05)$. This result suggests that the 
Table 3. Staining patterns for HLA class I and II in primary and metastatic SCC

\begin{tabular}{|c|c|c|c|c|c|c|c|c|c|c|c|c|c|}
\hline \multirow[b]{3}{*}{ Patient } & \multirow[b]{3}{*}{ Stage } & \multicolumn{4}{|c|}{ L368 mAb } & \multicolumn{4}{|c|}{$\mathrm{HC} 10 \mathrm{mAb}$} & \multicolumn{4}{|c|}{ LGIl-612.14 mAb } \\
\hline & & \multicolumn{2}{|c|}{ Primary } & \multicolumn{2}{|c|}{ Metastasis } & \multicolumn{2}{|c|}{ Primary } & \multicolumn{2}{|c|}{ Metastasis } & \multicolumn{2}{|c|}{ Primary } & \multicolumn{2}{|c|}{ Metastasis } \\
\hline & & Tumor & stroma & tumor & stroma & tumor & stroma & tumor & stroma & tumor & stroma & tumor & Stroma \\
\hline 1 & IIB & + & ++ & \pm & + & \pm & ++ & + & ++ & ++ & ++ & + & ++ \\
\hline 2 & IIB & \pm & \pm & \pm & \pm & + & ++ & - & ++ & \pm & ++ & - & ++ \\
\hline 3 & IIB & - & + & - & \pm & \pm & + & + & ++ & + & ++ & + & ++ \\
\hline 4 & IB & \pm & \pm & $\pm_{*}$ & + & \pm & \pm & \pm & ++ & \pm & + & \pm & + \\
\hline 5 & $\| A$ & \pm & \pm & $\mathrm{N}^{\star}$ & $\mathrm{N}$ & \pm & ++ & $\mathrm{N}$ & $\mathrm{N}$ & + & + & $\mathrm{N}$ & $\mathrm{N}$ \\
\hline 6 & IB & \pm & + & \pm & + & \pm & ++ & \pm & ++ & + & ++ & \pm & ++ \\
\hline 7 & IB & - & \pm & & \pm & \pm & ++ & \pm & ++ & \pm & + & \pm & ++ \\
\hline 8 & IIB & \pm & \pm & $\mathrm{N}$ & $\mathrm{N}$ & + & ++ & \pm & + & \pm & ++ & \pm & ++ \\
\hline 9 & IIB & + & ++ & - & \pm & + & ++ & \pm & + & \pm & ++ & \pm & ++ \\
\hline 10 & IB & \pm & + & \pm & + & + & ++ & \pm & ++ & \pm & ++ & \pm & ++ \\
\hline 11 & $\| A$ & \pm & \pm & & \pm & \pm & ++ & & \pm & \pm & ++ & \pm & ++ \\
\hline 12 & IIIB & + & + & \pm & \pm & + & ++ & + & ++ & \pm & ++ & \pm & ++ \\
\hline 13 & IB & - & \pm & - & \pm & + & ++ & - & ++ & \pm & ++ & - & ++ \\
\hline 14 & IB & \pm & \pm & \pm & \pm & ++ & ++ & - & \pm & \pm & ++ & - & ++ \\
\hline 15 & IIB & - & + & \pm & + & & + & \pm & + & \pm & ++ & \pm & ++ \\
\hline 16 & IIA & ++ & ++ & \pm & + & + & ++ & + & ++ & ++ & ++ & \pm & ++ \\
\hline 17 & IIB & \pm & \pm & \pm & ++ & \pm & ++ & \pm & ++ & \pm & ++ & \pm & ++ \\
\hline 18 & IIB & + & ++ & \pm & ++ & + & ++ & - & ++ & \pm & ++ & \pm & ++ \\
\hline 19 & IIA & \pm & ++ & \pm & + & - & \pm & - & \pm & - & ++ & - & \pm \\
\hline 20 & IIB & + & +++ & \pm & ++ & \pm & ++ & - & + & \pm & ++ & - & ++ \\
\hline 21 & IIIA & - & ++ & \pm & + & \pm & ++ & \pm & + & \pm & ++ & \pm & ++ \\
\hline 22 & IIB & ++ & ++ & + & + & ++ & ++ & \pm & + & ++ & ++ & + & ++ \\
\hline 23 & IB & + & + & + & ++ & \pm & ++ & \pm & ++ & + & ++ & \pm & ++ \\
\hline 24 & IIB & \pm & \pm & \pm & \pm & + & + & \pm & \pm & ++ & ++ & + & + \\
\hline 25 & IB & + & + & \pm & \pm & \pm & ++ & & ++ & + & ++ & \pm & ++ \\
\hline 26 & IIIB & + & ++ & \pm & + & \pm & + & + & + & - & ++ & \pm & + \\
\hline
\end{tabular}

*no metastasis.

Table 4. HLA class I and II expressions in invasive SCC

\begin{tabular}{|c|c|c|c|c|c|c|c|c|c|c|c|c|}
\hline & \multicolumn{4}{|c|}{ L368 mAb } & \multicolumn{4}{|c|}{$\mathrm{HC} 10 \mathrm{mAb}$} & \multicolumn{4}{|c|}{ LGII-612.14 mAb } \\
\hline & No. & Mean & Median & Range & No. & Mean & Median & Range & No. & Mean & Median & Range \\
\hline \multicolumn{13}{|c|}{ Primary lesion } \\
\hline Tumor & 26 & $2.269^{*}$ & 2 & $1-4$ & 26 & $2.423^{*}$ & 2 & $1-4$ & 26 & $2.423^{* *}$ & 2 & $1-4$ \\
\hline Stroma & 26 & 3 & 3 & $2-4$ & 26 & 3.692 & 4 & $2-4$ & 26 & 3.884 & 4 & $3-4$ \\
\hline \multicolumn{13}{|c|}{ Metastatic lesion } \\
\hline Tumor & 24 & $1.875^{\star \dagger}$ & 2 & $1-3$ & 25 & $1.84^{\star \dagger \ddagger}$ & 2 & $1-3$ & 25 & $1.96^{\star \dagger}$ & 2 & $1-3$ \\
\hline Stroma & 24 & 2.75 & 3 & $2-4$ & 25 & 3.44 & 4 & $2-4$ & 25 & 3.8 & 4 & $2-4$ \\
\hline
\end{tabular}

denotes significant difference between tumor and normal stroma in each lesion by Wilcoxon's signed rank test $(P<0.05)$.

${ }^{\dagger}$ denotes significant difference between primary and metastasis in each tissue by Wilcoxon's signed rank test $(P<0.05)$.

*denotes significant difference of the down-regulation rates from normal stroma to tumor compared to L368 in each lesion by Friedman test and Miller's nonparametric multiple comparison $(P<0.05)$.

degree of HLA class I and class II loss was not evident as disease progression in the early stage of adenocarcinomas (Figure 3A). Mixed expressions of weak positive and negative intensity are noted in both CIS and microinvasive lesions.

\section{Changes in HLA expression patterns according to} the progression of adenocarcinoma
HLA class I and II expression patterns in primary invasive adenocarcinoma tissues (Figure $3 \mathrm{~B}$ ) and metastatic lesions (Figure $3 \mathrm{C}$ ) were measured and made comparison with its corresponding normal stromal tissues on the same slide (Table 7).

Table 8 shows the degrees of HLA expressions according to HLA types in primary invasive and metastatic lesions expressed in mean, median and range from 

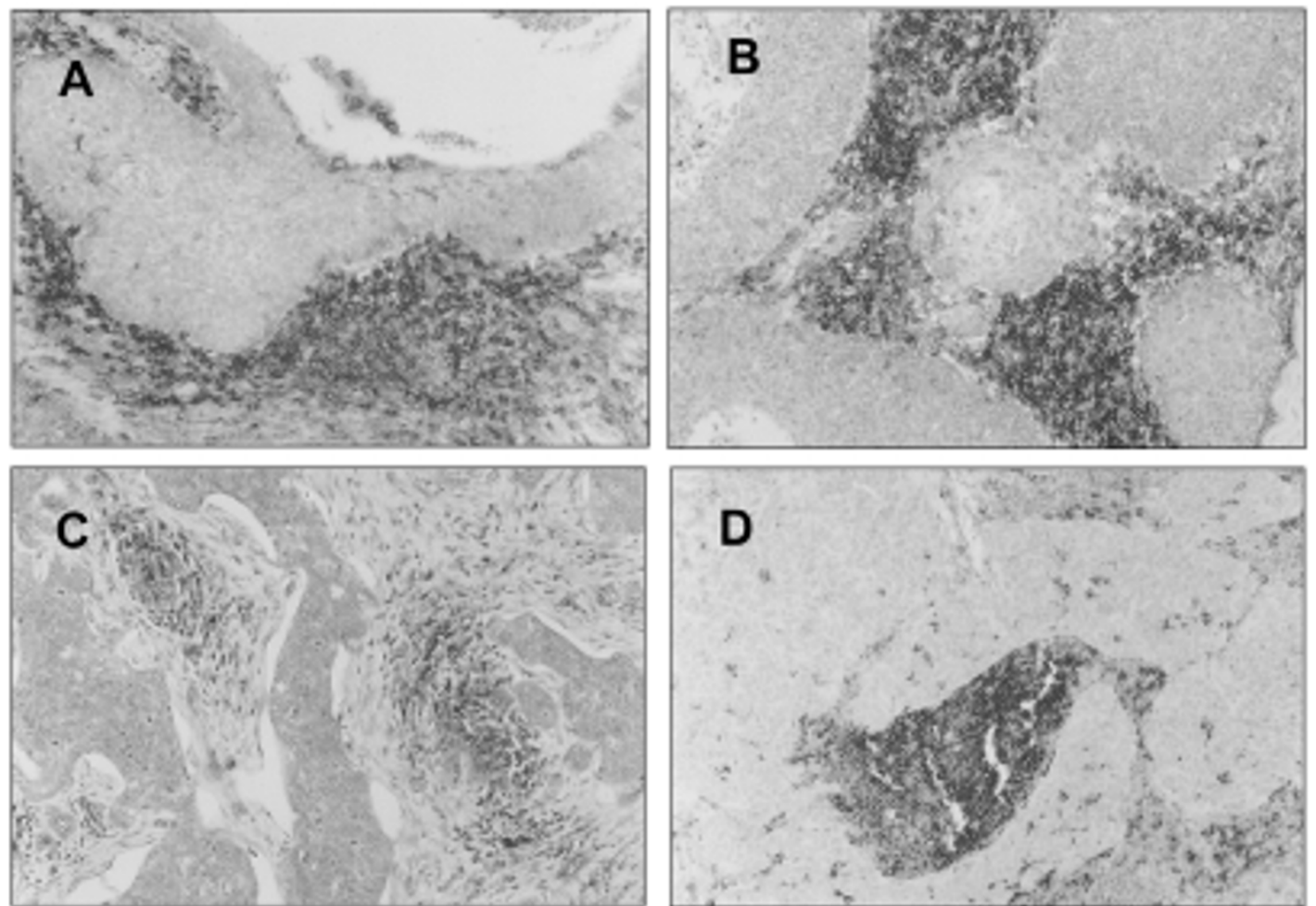

Figure 2. Immunohistochemical stainings of primary tumor and metastatic tissue sections of invasive SCC (large cell non-keratinizing). HLA class I expressions in primary (A) and metastatic (B) lesions. Immunostaining was performed by using a L368 mAb against HLA class I $\beta 2 \mathrm{~m}$. (A) The upper irregular island is moderately differentiated SCC. The tumor cells are large and rather pleomorphic nuclei, intercellular bridges are rarely seen and keratin pearls are absent. These tumor cells show weak positive intensity of HLA expression, but HLA antigens are strongly expressed in the area of lower normal stromal tissue. (Magnification 100). (B) Metastasized cells in the lymph node tissue from the same patient show weak positive intensity of expression in the central tumor area. Metastasized tumor lesion shows strong negative expression compared with the primary tumor lesion. But the surrounding normal stromal tissues show strong positive intensity of expression. (Magnificationx 100 ). HLA class II heavy chain expressions in primary tumor (C) and metastasized cells in the lymph node (D) of invasive SCC. Immunostaining was performed by using a LGII-612.14 mAb against HLA class II heavy chain. (C) This moderately differentiated and irregular shaped SCC nests show marked desmoplastic reaction (fibrosis). The tumor cells are negative and focally weak positive intensity of expression. And the fibrotic stroma between the cancer cell nests shows strong positive HLA expression. (Magnification $\times 100$ ). (D) The central stromal tissue shows strong positive intensity of expression and the surrounding metastatic tumor cells are negative intensity of expression. (Magnification $\times 100$ ).

Table 5. Staining patterns of HLA class I and II in early stage of adenocarcinoma

\begin{tabular}{|c|c|c|c|c|c|c|c|c|c|}
\hline \multirow[t]{2}{*}{ Patient } & \multicolumn{3}{|c|}{ L368 mAb } & \multicolumn{3}{|c|}{$\mathrm{HC10} \mathrm{mAb}$} & \multicolumn{3}{|c|}{ LGII-612.14 mAb } \\
\hline & MIC & $\mathrm{CIS}$ & Normal & MIC & CIS & Normal & MIC & $\mathrm{CIS}$ & Normal \\
\hline 1 & \pm & \pm & \pm & \pm & ++ & ++ & \pm & + & + \\
\hline 2 & \pm & \pm & \pm & \pm & + & + & - & \pm & \pm \\
\hline 3 & \pm & \pm & \pm & \pm & ++ & ++ & \pm & ++ & ++ \\
\hline
\end{tabular}

Table 6. HLA class I and II expressions in early stage of adenocarcinoma

\begin{tabular}{|c|c|c|c|c|c|c|c|c|c|c|c|c|}
\hline \multirow[t]{2}{*}{ Range } & \multicolumn{4}{|c|}{ L368 mAb } & \multicolumn{4}{|c|}{$\mathrm{HC} 10 \mathrm{mAb}$} & \multicolumn{4}{|c|}{ LGIl-612.14 mAb } \\
\hline & No & Mean & Median & Range & No & Mean & Median & Range & No & Mean & Median & Range \\
\hline MIC & 3 & 2 & 2 & $2-2$ & 3 & 2 & 2 & $2-2$ & 3 & 1.667 & 2 & $1-2$ \\
\hline CIS & 3 & 2 & 2 & $2-2$ & 3 & 3.667 & 4 & $3-4$ & 3 & 3 & 3 & $2-4$ \\
\hline Normal & 3 & 2 & 2 & $2-2$ & 3 & 3.667 & 4 & $3-4$ & 3 & 3 & 3 & $2-4$ \\
\hline
\end{tabular}

minimum to maximum values. In these invasive adenocarcinomas, only HLA-B, C heavy chains showed statistically significant down-regulation in primary invasive lesions compared to normal stromal tissues $(P=0.0158)$.
However, when the expressions were compared between primary and metastatic lesions, no statistically significant differences were seen in the HLA expression patterns between normal stromal and tumor tissues 

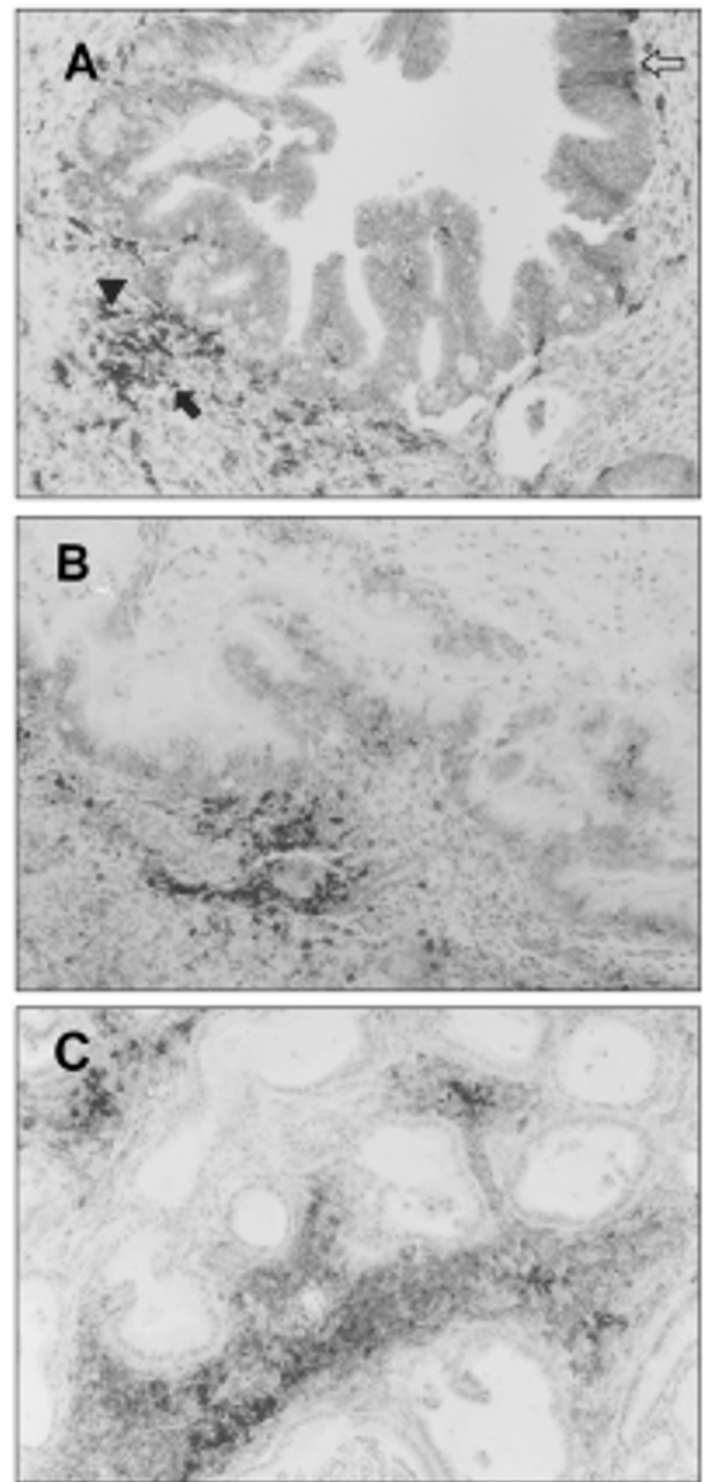

Figure 3. Immunohistochemical staining of early stage, primary invasive and metastatic adenocarcinoma of cervix. (A) Immunostaining was performed by using a HC-10 mAb against HLA class I B/C heavy chains. The cells of endocervical gland show decreased amount of cytoplasmic mucus, a high nucleo-cytoplasmic ratio and hyperchromatic nuclei. At the left portion in this figure, basement membrane disruption is shown subtly. In this lesion, mixed expressions of weak positive and negative intensity is noted (arrow head). At right adenocarcinoma in situ with intact basement membrane shows weak positive intensity of expression (open arrow). The left lower normal stromal tissue surrounding the glands shows strong positive intensity of expression (closed arrow). (Magnificationx100). (B) In this figure, upper levels are primary tumor lesion with weak positive intensity of expression and lower normal stromal tissues show strong positive intensity of expression. (Magnification $\times 100$ ). Immunostaining was performed by using a LGII-612.14 mAb against HLA class II heavy chains. (C) In the middle, normal stromal tissues in the metastatic lesion show strong positive intensity of expression. Metastasized tumor lesion shows strong negative expression compared with the primary tumor lesion (Magnification $\times 100$ ). Immunostaining was performed by using a LGII-612.14 mAb against HLA class II heavy chains.

within their corresponding primary and metastatic lesions, respectively (Table 8). When Millers nonparametric multiple comparison was performed to compare the HLA downregulation rates from normal stromal tissues to tumor tissues among HLA class I and II on the same slide, the down-regulation rate of HLA-B, $C$ heavy chain expression in tumor tissues of primary lesion compared to normal tissues was statistically significant than that of HLA class I $\beta 2 \mathrm{~m}(P<0.05)$. Also, on tumor tissues of metastatic lesion, compared to normal tissues, HLA class II heavy chain expression was statistically significantly downregulated than the down-regulation rate of $\beta 2 \mathrm{~m}(P<$ 0.05).

However, the rate of HLA-B, C heavy chain expression in primary lesions and that of HLA-B, C heavy chain expression in metastatic lesions did not show any difference compared to those in normal stromal tissues.

\section{Discussion}

The expressions of HLA class I and class II in CIS and microinvasive SCC were all decreased compared to normal cervical tissues in this study. In a few early lesions that showed a weak HLA expression, the infiltration of $\mathrm{T}$ - and $\mathrm{B}$-lymphocytes was observed more frequently than in areas with no HLA antigen expression. These results suggest that HLA antigens participate in the local immune response to early lesions, and that HLA expression may prevent tumor invasion similarly to its role in the early stage of SCC of the cervix.

Many researchers have reported that although genetic and morphologic changes occur in the preinvasive cancer stage at the beginning of tumor formation, they could not find a definite change in HLA class I expression (Glew et al., 1993; Hilders et al., 1994). However, if immune escape through HLA class I down-regulation is an important factor for tumor progression and invasion into the surrounding tissues (Raval et al., 1998), we could expect to detect tumor cells that do not express HLA class I antigen in CIS. Accordingly, the detection of the low frequency for HLA class I down-regulation in the previous studies might be due to low sensitivity of immunohistochemical staining used to detect HLA class I expression. With the development of mAbs that can detect the expression of a specific allele for HLA class $I$ and through a wide-range of studies using these developed mAbs, down-regulation of HLA class I might be seen in a high frequency even in preinvasive lesions.

In the present study, in CIS and MIC lesions of the cervix, the expressions of HLA class I $\beta 2 \mathrm{~m}$ and HLA-B, $C$ heavy chains and HLA class II heavy chain were characteristically decreased, suggesting that the downregulation of not only HLA class I but also HLA class II can be used as a marker for the early stage of SCC progressing into invasive tumors.

The expressions of HLA class I and class II antigen were all decreased in SCC of the cervix compared to 
Table 7. Staining patterns of HLA class I and II in primary and metastatic adenocarcinoma

\begin{tabular}{|c|c|c|c|c|c|c|c|c|c|c|c|c|c|}
\hline \multirow{3}{*}{ Patient } & \multirow{3}{*}{ Stage } & \multicolumn{4}{|c|}{ L368 mAb } & \multicolumn{4}{|c|}{$\mathrm{HC} 10 \mathrm{mAb}$} & \multicolumn{4}{|c|}{ LGII-612.14 mAb } \\
\hline & & \multicolumn{2}{|c|}{ Primary } & \multicolumn{2}{|c|}{ Metastasis } & \multicolumn{2}{|c|}{ Primary } & \multicolumn{2}{|c|}{ Metastasis } & \multicolumn{2}{|c|}{ Primary } & \multicolumn{2}{|c|}{ Metastasis } \\
\hline & & tumor & Stroma & Tumor & stroma & tumor & stroma & tumor & stroma & tumor & stroma & tumor & stroma \\
\hline 1 & IB & \pm & + & $\mathrm{N}^{*}$ & $\mathrm{~N}$ & \pm & ++ & $\mathrm{N}$ & $\mathrm{N}$ & ++ & ++ & $\mathrm{N}$ & $\mathrm{N}$ \\
\hline 2 & IB & - & + & $\mathrm{N}$ & $\mathrm{N}$ & \pm & ++ & $\mathrm{N}$ & $\mathrm{N}$ & \pm & ++ & $\mathrm{N}$ & $\mathrm{N}$ \\
\hline 3 & IB & ++ & \pm & $\mathrm{N}$ & $\mathrm{N}$ & \pm & + & $\mathrm{N}$ & $\mathrm{N}$ & ++ & ++ & $\mathrm{N}$ & $\mathrm{N}$ \\
\hline 4 & IB & \pm & \pm & \pm & + & \pm & \pm & ++ & + & + & ++ & - & + \\
\hline 5 & $\| A$ & \pm & \pm & \pm & - & ++ & ++ & \pm & ++ & ++ & ++ & - & ++ \\
\hline 6 & IIIC & \pm & \pm & \pm & + & \pm & ++ & - & ++ & \pm & ++ & - & ++ \\
\hline 7 & IV & \pm & \pm & $\mathrm{N}$ & $\mathrm{N}$ & - & ++ & $\mathrm{N}$ & $\mathrm{N}$ & + & ++ & $\mathrm{N}$ & $\mathrm{N}$ \\
\hline 8 & IB & \pm & \pm & \pm & - & ++ & ++ & \pm & ++ & ++ & ++ & - & ++ \\
\hline 9 & IIB & \pm & \pm & \pm & + & \pm & ++ & - & ++ & \pm & ++ & - & ++ \\
\hline
\end{tabular}

*no metastasis.

Table 8. HLA class I and II expressions in invasive adenocarcinomas

\begin{tabular}{|c|c|c|c|c|c|c|c|c|c|c|c|c|}
\hline & \multicolumn{4}{|c|}{ L368 mAb } & \multicolumn{4}{|c|}{$\mathrm{HC} 10 \mathrm{mAb}$} & \multicolumn{4}{|c|}{ LGII-612.14 mAb } \\
\hline & No. & Mean & Median & Range & No. & Mean & Median & Range & No. & Mean & Median & Range \\
\hline \multicolumn{13}{|c|}{ Primary lesion } \\
\hline Tumor & 9 & 2.111 & 2 & $1-4$ & 9 & $2.222^{\star \dagger}$ & 2 & 14 & 9 & 3.111 & 3 & $2-4$ \\
\hline Stroma & 9 & 2.222 & 2 & $2-3$ & 9 & 3.889 & 4 & 34 & 9 & 4 & 4 & $4-4$ \\
\hline \multicolumn{13}{|c|}{ Metastatic lesion } \\
\hline Tumor & 5 & 2 & 2 & 22 & 5 & 1.6 & 2 & 12 & 5 & $1 \dagger$ & 1 & $1-1$ \\
\hline Stroma & 5 & 2.2 & 3 & $1-3$ & 5 & 3.8 & 4 & 34 & 5 & 3.8 & 4 & $3-4$ \\
\hline
\end{tabular}

"denotes significant difference compared to normal epithelial tissue in each lesion by Wilcoxon's signed rank test $(P=0.0158)$.

${ }^{\dagger}$ denotes significant difference of the down-regulation rates from normal stroma to tumor compared to L368 in each lesion by Friedman test and Miller's nonparametric multiple comparison $(P<0.05)$.

those in normal cervical tissues, CIS and MIC, respectively, in this study.

In metastatic lesions from the same patient, the expressions of HLA class I $\beta 2 m$ and HLA-B, C heavy chains were somewhat decreased compared to those in primary lesions. And the expression of HLA class II heavy chain was further decreased than in primary tumors. The results from invasive SCC of the cervix that the expression of HLA class I antigen was further decreased in metastatic lymph node tissues compared to that in primary cervical cancer agreed with the result of the previous study (Cromme et al., 1994b). We also observed that the loss of HLA class I expression in SCC were more distinct than that of adenocarcinoma, showing that the pattern of HLA class I antigen loss is different according to histological types of cervical carcinoma.

In the present study, the results showed that HLA class II is expressed in normal cervical tissues, decreased in $\mathrm{CIS}$ and MIC lesions, and further decreased in primary invasive SCC tissues. Also, when the comparison was made in this SCC tissue with the metastatic lesion, the expression was significantly decreased in metastatic lesions. Although we could not explain precisely the significance of why the HLA class II expressions were decreased according to the tumor progression, through the fact that HLA class II molecule plays the role in antigen presentation and regulation of T-cell activation, we suppose that tumor cells suppress the expression of HLA class II to avoid the immune surveillance system, obtaining an advantageous characteristics in the tumor progression. Especially, when metastasis is present, the expression of HLA class II antigen was further decreased, which suggest that the degree of HLA class II antigen expression has a close correlation with tumor progression.

In this study, unlike in the case of CIS and microinvasive SCC, the degree of HLA class I and class II antigen loss was not evident with disease progression in the early stage of adenocarcinomas. In invasive adenocarcinoma of the cervix, the losses of HLA class I $\beta 2 \mathrm{~m}$, HLA-B, C heavy chains and HLA class II heavy chain showed an extremely low frequency compared to normal tissues, which were quite different from in case of invasive SCC. Although the expressions of HLA-B, C heavy chains were decreased significantly, the expressions of HLA class I $\beta 2 \mathrm{~m}$ and HLA class II heavy chain did not show any changes at all. In metastatic adenocarcinoma, no difference was seen in the expression pattern of HLA class I or HLA class II antigen compared to the degree of expression in the primary lesions.

With these results, we observed the characteristic 
down-regulation of HLA class I and HLA class II expressions in the progression of CIS to invasive cancer, showing the possibility that HLA class I and class II play an important role in SCC progression. However, we could not explain thoroughly whether each tumor characteristically shows different HLA expression patterns in the early stage of cancer, or what roles would HLA class I play, not in the early stage of cancer but after the tumor invades. In invasive SCC, the expressions of HLA class I $\beta 2 \mathrm{~m}, \mathrm{HLA}-\mathrm{B}, \mathrm{C}$ heavy chains and HLA class II were decreased compared to those in normal and preinvasive cancer stage and were down-regulated especially in metastatic SCC tissues in a very high frequency, shows that the down-regulation of HLA class I and class II antigen in SCC is one of the most important mechanisms when a tumor invades other adjacent tissues or when it goes through metastasis.

Further studies through using more tissue samples and comparing clinical variables are needed to determine whether HLA class I and II antigen expression could be used as a clinical marker of tumor progression and prognosis in cervical cancers.

\section{Acknowledgements}

This work was supported by Korea Research Foundation Grant (KRF-99-042-F00099).

\section{References}

Browning M, Dunnion D. HLA and cancer: implications for cancer immunotherapy and vaccination. Eur $\mathrm{J}$ Immunogenet 1997; 24:293-312

Campbell RD, Trowsdale J. Map of the human MHC. Immunol Today 1993;14:349-52

Cromme FV, Meijer CJ, Snijders PJ, Uyterlinde A, Kenemans P, Helmerhorst T, Stern PL, van den Brule AJ, Walboomers $\mathrm{JM}$. Analysis of MHC class I and II expression in relation to presence of HPV genotypes in premalignant and malignant cervical lesions. Br J Cancer 1993a;67:1372-80

Cromme FV, Snijders PJ, van den Brule AJ, Kenemans P, Meijer CJ, Walboomers JM. MHC class I expression in HPV 16 positive cervical carcinomas is post-transcriptionally controlled and independent from c-myc overexpression. Oncogene 1993b;8:2969-75

Cromme FV, Airey J, Heemels MT, Ploegh HL, Keating PJ, Stern PL, Meijer CJ, Walboomers JM. Loss of transporter protein, encoded by the TAP-1 gene, is highly correlated with loss of HLA expression in cervical carcinomas. J Exp Med 1994a;179:335-40

Cromme FV, van Bommel PF, Walboomers JM, Gallee MP, Stern PL, Kenemans P, Helmerhorst TJ, Stukart MJ, Meijer CJ. Differences in MHC and TAP-1 expression in cervical cancer lymph node metastases as compared with the primary tumours. Br J Cancer 1994b;69:1176-81

Garrido F, Cabrera T, Lopez-Nevot MA, Ruiz-Cabello F. HLA class I antigens in human tumors. Adv Cancer Res 1995;67: 155-95

Garrido F, Ruiz-Cabello F, Cabrera T, Perez-Villar JJ, LopezBotet M, Duggan-Keen M, Stern PL. Implications for immunosurveillance of altered HLA class I phenotypes in human tumors. Immunol Today 1997;18:89-95

Geertsen RC, Hofbauer GF, Yue FY, Manolio S, Burg G, Dummer R. Higher frequency of selective losses of HLA-A and $B$ allospecificities in metastasis than in primary melanoma lesions. J Invest Dermatol 1998;111:497-502

Glew SS, Connor ME, Snijders PJ, Stanbridge CM, Buckley $\mathrm{CH}$, Walboomers JM, Meijer CJ, Stern PL. HLA expression in pre-invasive cervical neoplasia in relation to human papilloma virus infection. Eur J Cancer 1993;29:1963-70

Hilders CG, Houbiers JG, Krul EJ, Fleuren GJ. The expression of histocompatibility-related leukocyte antigens in the pathway to cervical carcinoma. Am J Clin Pathol 1994; 101:512

Howard MC, Spack EG, Choudhury K, Greten TF, Schneck JP. MHC-based diagnostics and therapeutics. Immunol Today 1999;20:161-65

Hsu SM, Raine L, Fanger $\mathrm{H}$. The use of avidin-biotinperoxidase complex $(A B C)$ in immunoperoxidase technique: $A$ comparison between $A B C$ and unlabeled antibody (PAP) procedures. Histochem Cytochem 1981;29:577-80

Keating PJ, Cromme FV, Duggan-Keen M, Snijders PJ, Walboomers JM, Hunter RD, Dyer PA, Stern PL. Frequency of down-regulation of individual HLA-A and -B alleles in cervical carcinomas in relation to TAP-1 expression. $\mathrm{Br} \mathrm{J}$ Cancer 1995;72:405-11

Koopman LA, Mulder A, Corver WE, Anholts JD, Giphart MJ, Claas FH, Fleuren GJ. HLA class I phenotype and genotype alterations in cervical carcinomas and derivative cell lines. Tissue Antigens 1998;51:623-36

Powis SJ, Deverson EV, Coadwell WJ, Ciruela A, Huskisson NS, Smith H, Butcher GW, Howard JC. Effect of polymorphism of an MHC-linked transporter on the peptides assembled in a class I molecule. Nature 1992;357:211-15

Raval A, Puri N, Rath PC, Saxena RK. Cytokine regulation of expression of class I MHC antigens. Exp Mol Med 1998;30:1-13

Schrier PI, Bernards R, Vaessen RT, Houweling A, van der Eb AJ. Expression of class I major histocompatibility antigens switched off by highly oncogenic adenovirus 12 in transformed rat cells. Nature 1983;305:771-75

Temponi M, Kekish U, Hamby CV, Nielsen H, Marboe CC, Ferrone S. Characterization of anti-HLA class II monoclonal antibody LGII-612.14 reacting with formalin fixed tissues. J Immunol Methods 1993;161:239-56

Townsend A, Ohlen C, Bastin J, Ljunggren HG, Foster L, Karre K. Association of class I major histocompatibility heavy and light chains induced by viral peptides. Nature 1989;340: $443-48$ 